Pullen, I. M. \& Yellowlees, A. J. (1985) Is communication improving between general practitioners and psychiatrists? British Medical Journal, 290, 31-33.

P. NAIK

University Hospital

Alan S. LeE

Queen's Medical Centre

Nottingham NG7 2UH

\section{Role of general practitioners in the care of long-term} mentally ill patients

SIR: As the institutional care of psychiatric patients is replaced by community care, the role of GPs in the management of such patients is becoming increasingly prominent. Poynton \& Higgins (Journal, November $1991,159,703-706)$ correctly remark that it is timely to look at this role. It is salutary to note that Kendrick et al (1991) found among GPs an almost complete lack of specific practice policies for the care of long-term mentally ill patients.

A particular challenge to GPs is the care of schizophrenic patients, many of whom still have active symptomatology and profound social disabilities one year after discharge from mental hospital (Melzer et al, 1991). Recent literature concerning the community management of schizophrenia stresses the importance of integrating both pharmacological and psychosocial interventions through close co-operation between patients, carers, primary care professionals, and mental health specialists (e.g. Falloon et al, 1990).

In a recent (unpublished) study, I examined the part played by the GPs in one inner city practice in Sheffield in the care of the schizophrenic patients on their list. A sample of $\mathbf{4 7}$ patients was selected from a population of 72 known schizophrenics. Data were collected from computer records and practice notes, including details of antipsychotic medication, and a summary of correspondence and note entries for the two years between November 1989 and October 1991.

Of the 47 patients, 45 were receiving antipsychotic drugs from their GP. Of these, 28 were receiving a dose within the defined maintenance range specified by the British National Formulary (1991), and nine were receiving a higher dose. The latter figure may represent appropriate treatment of acute schizophrenic illness, or it may indicate over-prescription. Holloway (1988) found that the drug management of chronic mentally ill patients was frequently in the hands of GPs, and that over-prescription was common. He suggested local treatment protocols to improve prescribing practices.
GP liaison with other parties involved in patient care was assessed by analysing letters and phone calls filed or recorded in the notes during the two-year period. Correspondence was entered into regarding 28 of the 47 patients. For these 28, a mean of 5.5 letters were written or received (s.d. $=0.72$ ), of which over $90 \%$ were to or from psychiatrists. Telephone calls were recorded in the notes of 25 patients: a mean of 3.2 calls per patient (s.d. $=0.68$ ), which were distributed fairly evenly between psychiatrists, hostel staff, and other primary care professionals. A picture thus emerged of well developed communication with local psychiatrists, which was further enhanced by a fortnightly liaison-attachment scheme. Liaison with other involved parties was, however, haphazard and rare, furnishing little evidence of the teamwork favoured in the literature.

To assess the extent of GP involvement in patient care, all note entries made during the two-year period were analysed. A mean of $43.4($ s.d. $=3.16)$ entries were made per patient: approximately one entry for each patient every 17 days. More than half of these recorded the prescription of drugs or the administration of a depot injection - further evidence that the GPs were highly involved in managing patients' medication. Nearly $30 \%$ of entries referred to surgery attendances or home visits, showing the GPs to be in regular personal contact with their schizophrenic patients. Melzer et al (1991) similarly found high GP contact rates for schizophrenic patients, and suggest further work to examine the quality of that contact.

In summary, the GPs studied were highly involved in the care of their schizophrenic patients, playing a major role in the supervision of medication, and making regular personal contact through consultations. It is important to ensure the quality of both these aspects of involvement. Liaison with local psychiatrists was well developed, to the probable benefit of patient management, whereas liaison with other involved parties was poor. If the contribution of GPs is to be effectively integrated into a comprehensive community-based service, then a well developed system of liaison is of paramount importance.

Brittsh NAtional Formulary (1991) British National Formulary. London: British Medical Association and Royal Pharmaceutical Society of Great Britain.

Falloon, I. R. H., Shanahan, W., Laporta, M., et al (1990) Integrated family, general practice and mental health care in the management of schizophrenia. Journal of The Royal Society of Medicine, 83, 225-228.

Holloway, F. (1988) Prescribing for the long term mentally ill: a study of treatment practices. Family Practice, 5, 511-515.

Kendrick, T., SibBald, B., Burns, T., et al (1991) Role of general practitioners in the care of long term mentally ill patients. British Medical Journal, 302, 508-510. 
Melzer, D., Hale, A. S., Malik, S. J., et al (1991) Community care for patients with schizophrenia one year after hospital discharge. British Medical Journal. 303, 1023-1026.

University of Sheffield Medical School

JIM BODDINGTON

Beech Hill Road

Sheffield S10 2RX

\section{Developing training themes from HRH's delivery}

SIR: The Collegiate Trainees Committee welcome HRH The Prince of Wales's interest and depth of understanding in a thoughtful and provoking address to the College on its 150th anniversary (Journal, December 1991, 159, 763-768). The Committee wishes to develop some of the Prince's comments, particularly in relation to training.

We welcome HRH's emphasis on the holistic approach to healing and the need to emphasise the physical, mental and spiritual aspects of healing in the training of doctors in general and psychiatrists in particular. Religious and spiritual factors influence the experience and presentation of illness and a multi-ethnic perspective is essential in the management of psychiatric disorders in a multicultural society. This should be reflected in psychiatric training and, although many psychiatric units have close links with representatives of different religions and cultures, few psychiatric training schemes involve a formal input.

In the current climate of health care in Britain, we are concerned about diminishing opportunities for psychiatric trainees to learn from the specialist skills of other allied professionals, mainly as a result of reduced numbers of psychologists, social workers, and occupational therapists in psychiatric services. We welcome the Prince's comments on closer liaison between health and social services and see such joint work and training as helpful in developing a more holistic approach.

HRH highlighted the division between biologically orientated psychiatrists who were over-reliant on physical and chemical modes of treatment, and psychotherapists who solely use psychological treatments. We think it is important that such an impression must be seen against a backdrop of limited resources - especially for the development of psychotherapeutic treatments within the National Health Service. Good training enables an eclectic approach, but poor training and time restraints may lead to more 'medication focused' consultations, hence further distancing the psychiatrist and his or her patient from an holistic approach.

HRH identified the lack of public exposure to mental illness in the media. We consider that the potential for misportrayal of mental health issues and psychiatric practice exceeds that of many other branches of medicine. We feel that psychiatrists as a profession should be prepared to be more active in media liaison and we are concerned that presentation skills, from conducting a case conference through to lecturing and media skills, have been largely ignored in the training of psychiatrists. We understand that the Public Education Committee of the College is attempting to address some of these problems by teaching psychiatrists how to talk to the media.

HRH brought attention to several areas of changing service provision which have important implications for psychiatric training. With increasing numbers of chronically ill patients being discharged from long-term beds and fewer beds being available for acute care, an increase in emergency presentations to psychiatric hospitals, accident and emergency departments and police services is to be expected (Department of Health, 1989). Emphasis thus needs to be given in training on how to manage psychiatric emergency consultations and, in planning such services, the supervision and safety of trainees who man them is a priority. Psychiatric training must address the changing needs of the community and its health service. Community psychiatry, liaison with general practitioners, closer work with social work departments, and the voluntary sector all require greater attention. Closer training links between general practitioners and psychiatrists will be of help, and such links should be reciprocal. We would also encourage trainees to visit and learn about models of service practised in other areas of the country and beyond.

We hope that psychiatrists will not ignore the challenges put forward by HRH's address.

Department of Health (1989) Caring for People. London: HMSO.

R. KEHOE
A. MOORE
J. PEARCE
M. WARD
F. JAMIESON
J. QUINN
Working Group, Collegiate Trainees Committee
The Royal College of Psychiatrists
17 Belgrave Square
London SWIX $8 P G$

SIR: I would like to add some comments to the wise remarks of the HRH The Prince of Wales (Journal, December 1991, 159, 763-768) regarding the current and future roles of psychiatrists. Most of us are aware of the biopsychosocial theoretical framework 